\title{
Serum Alpha-(1,3)-Fucosyltransferase IV, Copper, and Zinc Levels in Patients with Colorectal Cancer and Healthy Controls
}

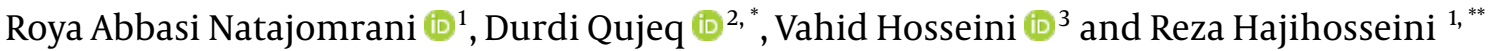 \\ ${ }^{1}$ Department of Biology, Payame Noor University, Tehran, Iran \\ ${ }^{2}$ Cellular and Molecular Biology Research Center (CMBRC), Health Research Institute, Babol University of Medical Sciences, Babol, Iran \\ ${ }^{3}$ Gut and Liver Research Center, Mazandaran University of Medical Sciences, Sari, Iran \\ "Corresponding author: Department of Clinical Biochemistry, Faculty of Medicine, Babol University of Medical Sciences, Babol, Iran. Email: dqujeq@gmail.com \\ ${ }^{* *}$ Corresponding author: Department of Biology, Payame Noor University, Tehran, Iran. Email: hosseini@pnu.ac.ir
}

Received 2020 June 30; Revised 2020 November 02; Accepted 2020 November 21.

\begin{abstract}
Background: Colorectal cancer (CRC) has no significant clinical symptoms at the early stages, and the molecular differences in the serum of the patients and healthy subjects could be assessed to identify the biological markers that indicate the detection of this cancer at the levels of the biological system.

Objectives: The present study aimed to assess the concentrations of the alpha-(1,3)-fucosyltransferase IV(FUT4) enzyme and copper (Cu) and zinc ( $\mathrm{Zn})$ as biological elements.

Methods: This case-control study was conducted on 40 patients with CRC, including 20 men and 20 women. A metal-free sterile tube was used to collect five milliliters of venous blood. The enzyme-linked immunosorbent assay (ELISA) based on the Biotin doubleantibody sandwich technology was used to measure the human FUT4 in the sera. In addition, plasma zinc and copper values were determined using Zist Chem Diagnostics kits.

Results: The mean FUT4 levels in the CRC patients was slightly higher than the control group $(\mathrm{P}=0.17)$, and the mean serum copper and zinc levels of these patients were lower than the control group with a significance difference in this regard $(P<0.001)$. In addition, the AUC of FUT4, copper, and zinc was $0.58,0.80$, and 0.77 , respectively.

Conclusions: According to the results, the altered levels of FUT4, copper, and zinc in the serum of the CRC patients compared to the healthy controls could be an indicator associated with the CRC disease course. Furthermore, the unusual changes in the FUT4, copper, and zinc levels may signify CRC development, which plays a key role in the diagnosis and monitoring of this cancer.
\end{abstract}

Keywords: Alpha-(1,3)-Fucosyltransferase IV, Colorectal Cancer, Copper, Zinc

\section{Background}

Colon cancer is the third most common cancer in the world that could lead to death (1). Colorectal cancer (CRC) is the most prevalent cancer in Iranian men and women (2), which is caused by the uncontrolled growth of the epithelial cells of the colon and rectum layers of the gastrointestinal system (3). Attempts have been made to identify new biomarkers or a set of biological indicators that are prestigious and essential to clinical trials for the early diagnosis of CRC (4).

Currently, studies are focused on various proteins, glycoproteins, and cellular and humeral substances that are involved in the homeostasis mechanisms of the cells as potential cancer markers (5), and several markers have been established for cancer detection $(6,7)$. Recently, researchers have investigated the DNA damage response in cancer and some of the influential factors in the patho- genesis of human diseases $(8,9)$. Growing evidence supports the measurement of specific biomarkers in the biological samples of cancer patients $(10,11)$. Furthermore, researchers have suggested that the regulatory functions of circular RNAs and targeting the mammalian target of rapamycin signaling could be considered in cancer treatment $(12,13)$. The findings in this regard also indicate that the CRISPR/Cas9 technology could be used to recognize disease pathogenesis (14).

Cellular glycosylation changes are important during the embryonic development and cellular activation and are regulated by glycosyltransferases enzymes (15). Unusual glycosylation is considered to be a sign of malignant cellular development, which plays a key role in the development of this mutation (16).

Fucosyltransferases are the enzymes that are involved in the addition of fucose to glycan structures (17). One type

Copyright (c) 2020, Journal of Kermanshah University of Medical Sciences. This is an open-access article distributed under the terms of the Creative Commons Attribution-NonCommercial 4.0 International License (http://creativecommons.org/licenses/by-nc/4.0/) which permits copy and redistribute the material just in noncommercial usages, provided the original work is properly cited. 
of fucosyltransferase enzymes are expressed in the cells and have been shown to be responsible for generating various glycosylated bonds, which are essential to synthesis of Lewis antigens and transfer fucose residues to glycoprotein cells (18). In addition, the high expression of the fucosylated antigens associated with fucosyltransferase has been reported in colon cancer (19). Fucosyltransferase IV (FUT4) is an effective biomarker of breast cancer diagnosis (20). Previous findings have indicated that the function of tumor suppressants in the prevention of CRC progression is through the regulation of FUT4 (21).

Elements are crucial nutrients for the activity of antioxidant enzymes in several biological processes (22). Zinc $(\mathrm{Zn})$ and copper $(\mathrm{Cu})$ are two biological elements that are significantly involved in the activity of diverse enzymes (23). Researchers have claimed that copper plays a pivotal role in angiogenesis, which causes tumor, invasion, and metastasis, and zinc is essential to the immune function (24-26). The association between oxidative stress and glycobiology has been investigated, as well as the changes in the structure of glycans in response to the changes in the redox system. Accordingly, the imbalance of oxidants and antioxidant enzymes leads to oxidative stress, which is involved in the expression of glycosyltransferase genes and causes structural and functional changes in glycoproteins, thereby increasing the risk of chronic diseases such as cancer (27).

\section{Objectives}

The present study aimed to assess the concentrations of the FUT4 enzyme and copper and zinc as biological elements in order to determine the difference between these factors in CRC patients and healthy subjects, as well as the associations of these factors with the identification of the mechanisms involved in the development of CRC.

\section{Methods}

\subsection{Patients and Samples}

This case-control study was conducted on 40 CRC patients (20 men and 20 women), who were admitted to Imam Khomeini Teaching Hospital and Tuba Clinic, affiliated to Mazandaran University of Medical Sciences in Sari, north of Iran, during November 2017-January 2019. The patients were aged 30 - 70 years, and the CRC diagnosis was confirmed based on the pathology report. The control group consisted of 40 healthy volunteers ( 8 males and 32 females) aged 30 - 70 years. To reduce and control the effects of confounders, the selection of the study and control groups was matched to the age range, region, and demographic characteristics.

A metal-free sterile tube was used to collect five milliliters of venous blood, and the sample was put to a clot. Afterwards, serum extraction was performed via centrifugation at 3,000 rpm for 10 minutes. The serum samples with no indication of hemolysis were aliquot into Eppendorf tubes and preserved at the temperature of $-80^{\circ} \mathrm{C}$ before assaying.

\subsection{Inclusion Criteria}

The inclusion criteria of the study were age of $30-70$ years, CRC patients not diagnosed based on consultation with a gastroenterologist, and receiving no chemotherapy and radiotherapy treatments at the time of the study.

\subsection{Exclusion Criteria}

The exclusion criteria were patients aged more than 70 and less than 30 years, patients undergoing chemotherapy and radiotherapy, smoking habits, and history of other cancers and malignant/autoimmune diseases.

In the present study, ELISA based on the Biotin doubleantibody sandwich technology was used to measure the human FUT4 in the sera. Prior to the assay and to defrost the serum samples thoroughly, they were maintained at room temperature for roughly 30 minutes. The human FUT4 enzyme-linked immunosorbent assay kit (Bioassay Technology Laboratory, E1951Hu) was used to specify the FUT4 in duplicate. The assays were carried out in accordance with the instructions of the manufacturer.

After preparing five dilutions of the basic standard, 50 microliters of the standard solution were added to the standard solution well. In addition, 40 microliters of the sample and 10 microliters of the FUT4 antibodies were added to the sample well, and only 50 microliters of chromogens and stop solution were added to the blank well. Following that, the wells were gently shaken to be mixed and incubated at the temperature of $37^{\circ} \mathrm{C}$ for 60 minutes.

After the preparation of the washing solution, each well was filled with the solution and drained after $30 \mathrm{sec}$ onds standing; the procedure was repeated five times and the plate was also blotted. For color development, $50 \mathrm{mi}-$ croliters of the chromogenic solution A and B was added to each well, and after shaking, they were incubated at the temperature of $37^{\circ} \mathrm{C}$ for 10 minutes. At the next stage, 50 microliters of the stop solution was added to each well to discontinue the reaction.

An ELISA reader (Awareness Technology INC Star Fax2100) was used to read the data at the wavelength of 450 nanometers. The FUT4 concentrations were specified through estimation based on the standard curve developed by plotting the absorbance of the standard and 
matching concentrations. In addition, the plasma zinc and copper values were determined using Zist Chem Diagnostics kits (REF10-517) and Biorexfars-Copper-Bxc0341, Iran. The assays were performed in accordance with the instructions of the manufacturers, and the absorbance was read at 578 nanometers using a spectrophotometer (UNICO-AJU1104001).

Written informed consent was obtained from the patients prior to the study. The study protocol was approved by the Human Subjects Ethics Board of Payame Noor University (Iran), and the research was conducted in accordance with the Helsinki Declaration of 1975 (revised in 2013). All the protocols involving the patients and control subjects were also approved by the Ethics Committee of Payame Noor University (code: IR.PNU.REC.1397.036).

\subsection{Statistical Analysis}

Data analysis was performed in SPSS version 21, and the data were expressed as mean and standard deviation (SD). T-test and Mann-Whitney U test were applied to compare the CRC patients with the control group in terms of the serum FUT4, zinc, and copper levels. In addition, Spearman's rank correlations were used for the correlation analysis between the quantitative variables. The receiver operating characteristic (ROC) curve analysis was also performed to determine the significant associations of FUT4, zinc, and copper and assess the diagnostic performance for CRC. In all the statistical analyses, the P-value of less than 0.05 was considered significant.

\section{Results}

Table 1 shows the demographic characteristics of the CRC patients and healthy controls. The mean age of the CRC patients was $60 \pm 9.42$ years (range: 30 - 70 years), and the mean age of the healthy controls was $43 \pm 13.4$ years (range: 30 - 70 years). Table 2 and Figure 1 show the FUT4, copper, and zinc serum levels in the CRC patients and healthy controls.

According to the findings, the mean FUT4 level of the CRC patients was $1.01 \pm 1.9 \mathrm{ng} / \mathrm{mL}$, which was slightly higher than the mean FUT4 level of the healthy controls $(0.5 \pm 0.64 \mathrm{ng} / \mathrm{mL})$. The serum concentrations of zinc and copper reduced significantly in the CRC patients compared to the healthy controls $(\mathrm{P}<0.001)$. The mean copper level was $83.9 \pm 15.61$ and $101.4 \pm 15.69 \mu \mathrm{g} / \mathrm{dL}$ in the CRC patients and healthy controls, respectively. The mean zinc level was $77.44 \pm 18.09$ and $94.6 \pm 15.35 \mu \mathrm{g} / \mathrm{dL}$ in the CRC patients and healthy controls, respectively. Table 3 shows the Pearson's correlation-coefficient ( $r$ ) and P-value of the FUT4, copper,

\begin{tabular}{lcc}
\hline \multicolumn{2}{l}{ Table 1. Demographic Characteristics of CRC Patients and Healthy Controls ${ }^{\mathrm{a}}$} \\
\hline & Healthy Controls, $\mathbf{N}=\mathbf{4 0}$ & CRC Patients, $\mathbf{N}=\mathbf{4 0}$ \\
\hline Gender, No. (\%) & $32(80)$ & $20(50)$ \\
Female & $8(20)$ & $20(50)$ \\
\hline Male & $43 \pm 13.4$ & $60 \pm 9.42$ \\
Age $(\mathbf{y})$ & $69.38 \pm 8.16$ & $73.32 \pm 11.63$ \\
Weight $(\mathbf{k g})$ & $164.48 \pm 6.9$ & $164.24 \pm 7.21$ \\
Height $(\mathbf{c m})$ & $25.61 \pm 2.31$ & $27.22 \pm 4.3$ \\
\hline BMI $\left(\mathbf{k g} / \mathbf{m}^{2}\right)$ &
\end{tabular}

${ }^{\mathrm{a}}$ Values are expressed as mean \pm SD unless otherwise indicated.

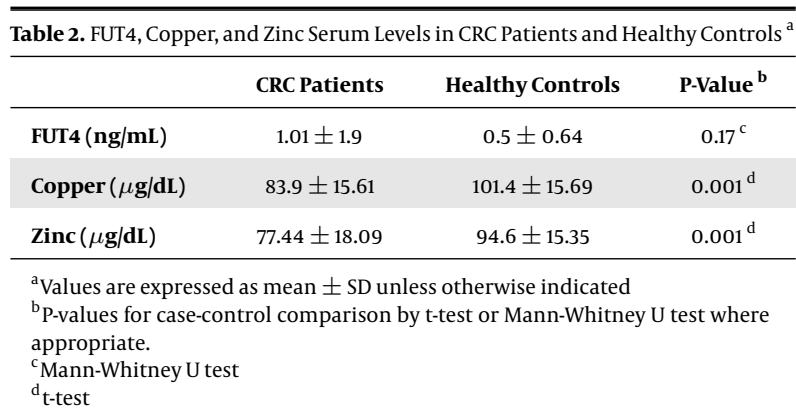

and zinc serum levels in the CRC patients and healthy controls. Notably, the area under curve (AUC) was used to express the results of the ROC curve analysis (Figures 2 and 3). The AUC values of FUT4, copper, and zinc were $0.58,0.80$, and 0.77 , respectively.

Table 3. Pearson's Correlation-coefficients ( $r$ ) and P-values between FUT4, Copper, and Zinc Serum Levels in CRC Patients a Healthy Controls

\begin{tabular}{lcc}
\hline Correlation-Coefficients & $\mathbf{r}$ & $\mathbf{r}$ \\
\hline Serum FUT4 and Serum Copper & -0.049 & 0.252 \\
Serum FUT4 and Serum Zinc & -0.115 & -0.09 \\
\hline
\end{tabular}

\section{Discussion}

The main findings of the present study were the increased FUT4 level and decreased copper and zinc levels in the serum of the CRC patients compared to the healthy controls. The slight increase in the level of FUT4 in CRC patients might accelerate the disease process through the fucosylation of circulating compounds. On the other hand, altered serum levels of $\alpha 1$, 3-fucosylation may be associated with the process of CRC. In this regard, fucosylation might be altered by the zinc and copper status since these elements have unique properties in biological processes. Therefore, these elements may affect the changes in the level of the FUT4 enzyme in CRC patients. 

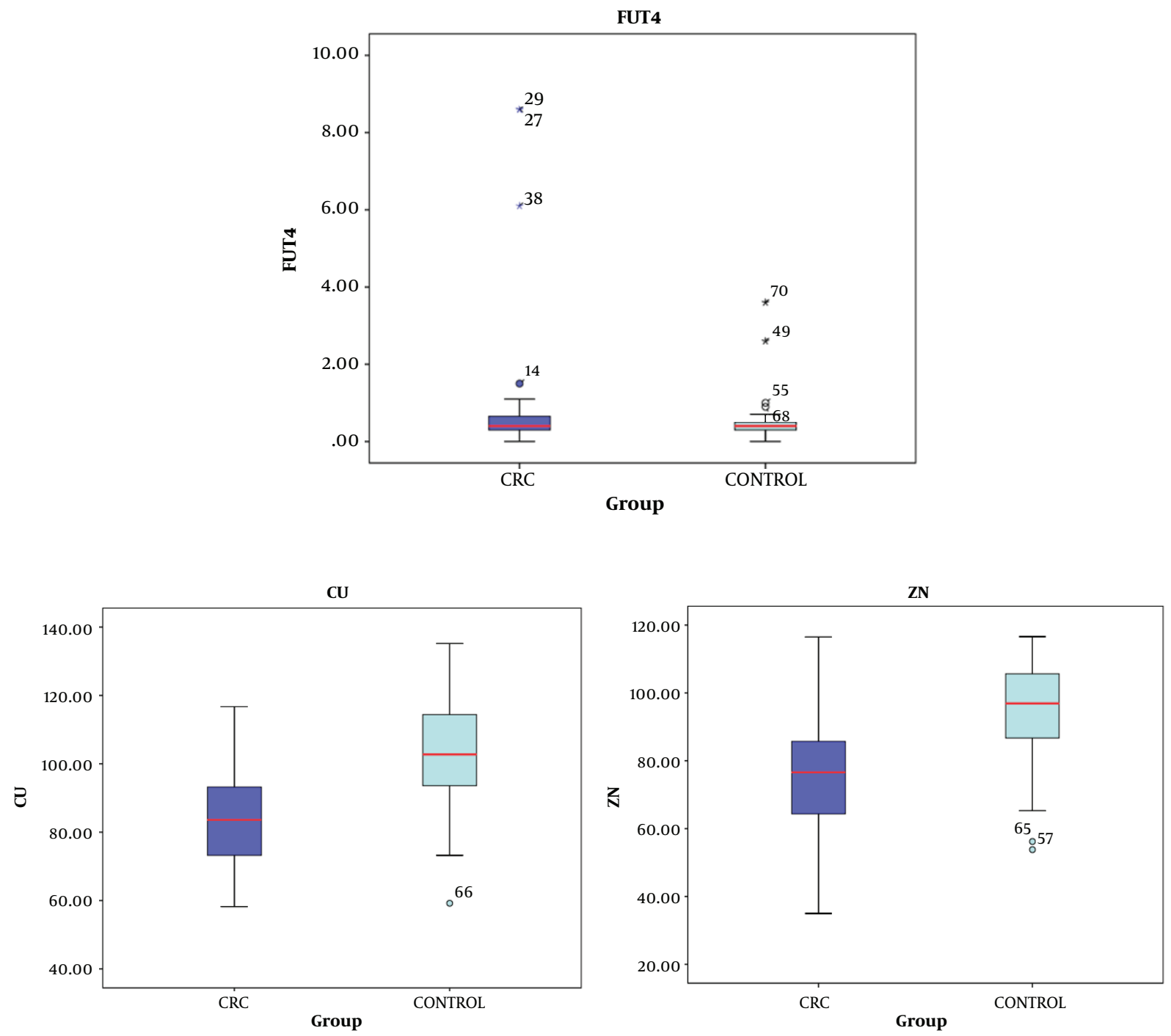

Figure 1. Levels of FUT4, Copper (Cu), and Zinc (Zn) in Patients with CRC and Healthy Controls Based on ELSIA and Spectrophotometric Method, respectively; A) FUT4, B) Cu, C) $\mathrm{Zn}$ (t-test or Mann-Whitney U test used to compare to respective normal group where appropriate.)

Fucosyltransferases are key enzymes that accelerate the process of malignancy through the fucosylation of cellular compounds $(28,29)$. In a study in this regard, Laura Muinelo-Romay et al. analyzed the enzyme activity of alpha-(1, 6)-fucosyltransferase in normal and CRC tissues, reporting a significant increase in the expression of alpha-(1, 6)-fucosyltransferase in the tumor tissues compared to the normal and transitional tissues, inflammatory lesions, and adenomas (30). Furthermore, researchers have demonstrated that the expression of alpha- $(1,6)$ fucosyltransferase may be a good prognostic factor for the prediction of CRC (31).

According to the ROC analysis results in the present study (Figure 2), the AUC of FUT4 was 0.58. Notably, FUT4 is an effective biomarker of breast cancer diagnosis (20). Our findings in this regard are inconsistent with the results of other studies. The slight increase in the serum levels of FUT4 may be attributed to the differences in the cancer type, various stages of the disease, and number of the tested samples in other studies (20, 28-31). For instance, Taniguchi et al. reported that the expression of glycan is altered by cellular redox states, suggesting that key redox- 


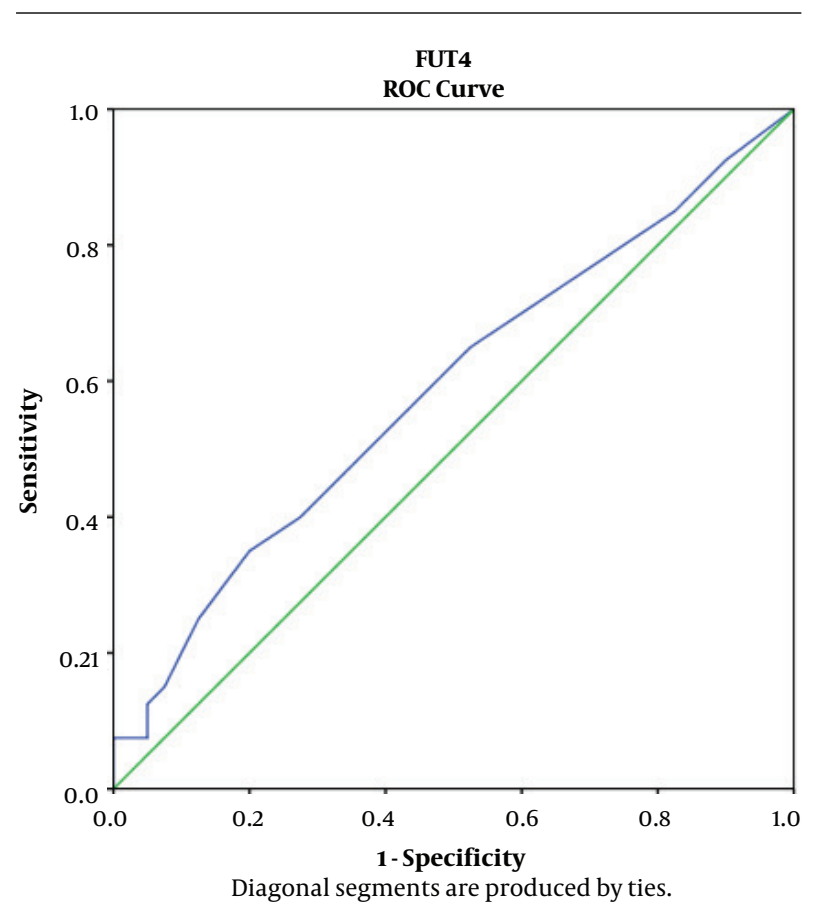

Figure 2. Analysis of FUT4, $\mathrm{Cu}$, and $\mathrm{Zn}$ of Sensitivity and Specificity in CRC Diagnosis by ROC (Data of ELISA for FUT4 [larger test result indicated more positive tests and spectrophotometric method for $\mathrm{Cu}$ and $\mathrm{Zn}$ [smaller test results indicated more positive tests])

related transcription factors are involved in the transcriptional activation of some glycosyltransferase genes (27).

In the present study, the serum levels of copper and zinc were lower in the CRC patients compared to the healthy controls, with a significant difference in this regard. With respect to the ROC analysis results (Figure 3), the AUC of the copper and zinc levels were AUC $=0.80$ and AUC $=0.77$, respectively). Therefore, it could be inferred that copper and zinc deficiencies may be involved in the development of CRC. In this regard, Nora et al. reported that compared to healthy controls, colon cancer patients were not different in terms of copper concentrations, while zinc concentrations were significantly lower in the colon cancer patients compared to the normal subjects (32). Furthermore, a recent study conducted by Sohrabi et al. on cancerous and adjacent non-cancerous tissues with CRC indicated that the median levels of zinc and copper in the cancerous tissues were significantly higher than the healthy tissues, which is inconsistent with our findings. Changes in zinc levels may be associated with the movement of circulating zinc to the colon cancer tissues and its involvement in the function of the antioxidant enzymes to relieve oxidative stress (22). In the study performed by Khoshdel et al. on patients with CRC, the total mean serum cop-

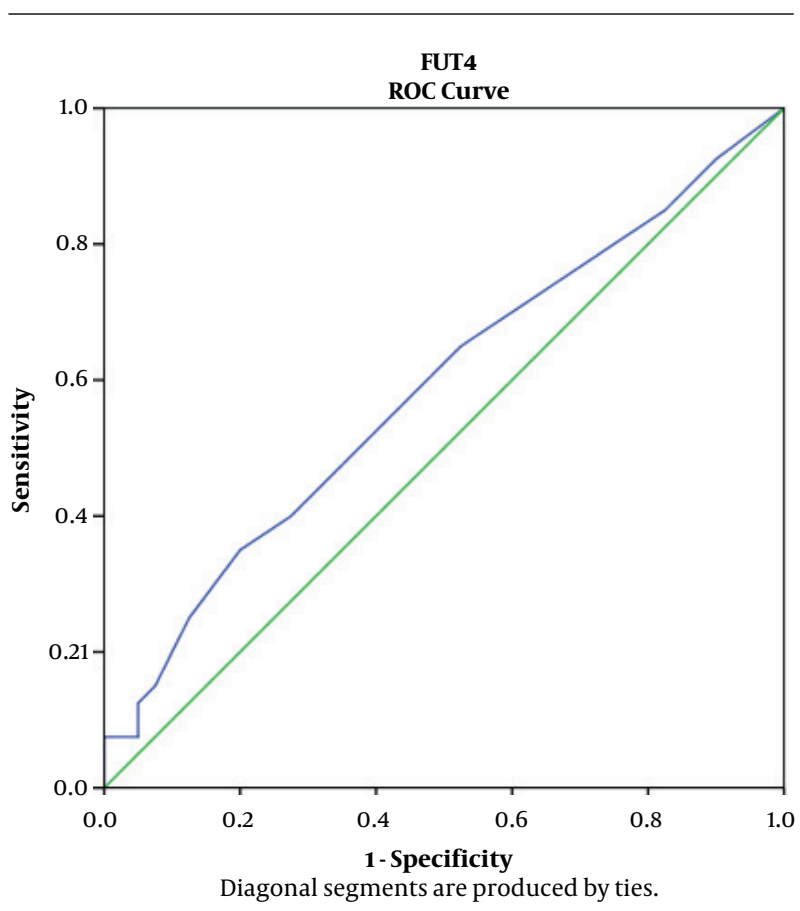

Figure 3. Analysis $\mathrm{CU}, \mathrm{ZN}$ of sensitivity and specificity in the diagnosis of $\mathrm{CRC}$ by ROC curve. Data from spectrophotometric method for CU and ZN (considering the smaller test result indicates more positive test). AUC $(\mathrm{CU}=0.8)(\mathrm{ZN}=0.77)$, AUC $(0.5$ to 0.6 ) bad, (0.6 to 0.8 ) fair, (0.8 to 0.9 ) good, 0.9 to 1 ) very good or excellent classifer.

per and zinc concentrations in the CRC patients reduced significantly compared to the control group (24). On the other hand, a cohort investigation by Stepien et al. demonstrated that the higher circulating concentration of copper was associated with the development of CRC. This is inconsistent with the results of the present study, and the discrepancy could be due to the increased levels of the proinflammatory cytokines that stimulate the hepatic synthesis of the acute-phase reactive protein ceruloplasmin, which is a major copper transporter (33). Epidemiological evidence also suggests that altered levels of trace elements are associated with fatal diseases, such as cancer. Previous findings have confirmed the altered concentration of zinc and copper in cancer patients (24). However, the findings regarding the increased/decreased levels of copper and zinc in CRC patients are conflicting, which could be due to the differences in the study design, sample sizes, and other environmental factors.

\subsection{Limitations of the Study}

One of the limitations of the current research was the small sample size, which might have decreased the power of the statistical analysis of the correlation between the FUT4, zinc, and copper serum levels in the patients. In addition, no data was available on the stage of CRC. 


\subsection{Conclusion}

According to the results, the CRC patients had slightly higher FUT4 levels compared to the healthy controls, while the serum concentrations of zinc and copper were significantly lower in the CRC patients compared to the healthy subjects. These factors are involved intercellular and intracellular biological processes. Therefore, it could be inferred that copper and zinc deficiencies and the slight increase in FUT4 may be involved in the development of CRC.

\section{Acknowledgments}

Hereby, we extend our gratitude to the staff of Imam Khomeini Teaching Hospital and Tuba Clinic, affiliated to Mazandaran University of Medical Sciences in Sari, Iran for assisting us in this research project.

\section{Footnotes}

Authors' Contribution: D. Q. and R. H. designed the experiments; R. A. N. O. and V.H. performed the experiments; R. A. N. O. analyzed the data and drafted the manuscript.

Conflict of Interests: None declared.

Ethical Approval: All the protocols involving the patients and control subjects were confirmed by the Ethics Committee of Payame Noor University (code: IR.PNU.REC.1397.036).

Funding/Support: This study was financially supported by the Department of Biochemistry at the Faculty of Sciences of Payame Noor University in Tehran, Iran as a PhD dissertation (No. 2-1396/10/26).

Informed Consent: Written informed consent was obtained from the patients prior to the study.

\section{References}

1. Das V, Kalita J, Pal M. Predictive and prognostic biomarkers in colorectal cancer: A systematic review of recent advances and challenges. Biomed Pharmacother. 2017;87:8-19. doi: 10.1016/j.biopha.2016.12.064. [PubMed: 28040600]

2. Janbabaei G, Hedayatizadeh-Omran A, Alizadeh-Navaei R, Moradi S, Ahmadi A, Alashti MR, et al. An epidemiological study on patients with colorectal cancer admitted to one referral center in north of Iran from 2006 to 2015. IIC. 2017;3:0.5.

3. Bresalier RS. Colorectal cancer. Sleisenger and Fordtran's gastrointestinal and liver disease. Saunders; 2016. p. 2248-96.

4. Kuipers EJ, Grady WM, Lieberman D, Seufferlein T, Sung JJ, Boelens PG, et al. Colorectal cancer. Nat Rev Dis Primers. 2015;1:15065. doi: 10.1038/nrdp.2015.65. [PubMed: 27189416]. [PubMed Central: PMC4874655].

5. Armitage EG, Barbas C. Metabolomics in cancer biomarker discovery: current trends and future perspectives. J Pharm Biomed Anal. 2014;87:1-11. doi:10.1016/j.jpba.2013.08.041. [PubMed: 24091079].

6. Mahmoodi A, Qujeq D, Danesh doust D, Karimi M. Determination of serum survivin for prognostic role in esophageal cancer. Int J Res Appl Basic Med Sci. 2020;6(1):9-13.
7. Aghcheli K, Parsian H, Qujeq D, Talebi M, Mosapour A, Khalilipour E, et al. Serum hyaluronic acid and laminin as potential tumor markers for upper gastrointestinal cancers. Eur J Intern Med. 2012;23(1):58-64 doi: 10.1016/j.ejim.2011.07.018. [PubMed: 22153533].

8. Samavarchi Tehrani S, Mahmoodzadeh Hosseini H, Yousefi T, Abolghasemi M, Qujeq D, Maniati M, et al. The crosstalk between trace elements with DNA damage response, repair, and oxidative stress in cancer. J Cell Biochem. 2018;120(2). doi: 10.1002/jcb.27617. [PubMed: 30378148].

9. Nejat Pish-Kenari F, Qujeq D, Maghsoudi H. Some of the effective factors in the pathogenesis of gastro-oesophageal reflux disease. $J$ Cell Mol Med. 2018;22(12):6401-4. doi: 10.1111/jcmm.13939. [PubMed: 30320456]. [PubMed Central: PMC6237569].

10. Abolghasemi M, Yousefi T, Maniati M, Qujeq D. The interplay of Klotho with signaling pathway and microRNAs in cancers. J Cell Biochem. 2019;120(9):14306-17. doi:10.1002/jcb.29022. [PubMed: 31127658].

11. Yousefi M, Qujeq D, Shafi H, Tilaki KH. Serum and urine levels of sarcosine in benign prostatic hyperplasia and newly diagnosed prostate cancer patients. J Kermanshah Univ Med Sci. 2020;24(1). doi 10.5812/jkums.97000.

12. Tamaddoni A, Mohammadi E, Sedaghat F, Qujeq D, As'Habi A. The anticancer effects of curcumin via targeting the mammalian target of rapamycin complex 1 (mTORC1) signaling pathway. Pharmacol Res. 2020;156:104798. doi: 10.1016/j.phrs.2020.104798. [PubMed 32278045].

13. Soghli N, Qujeq D, Yousefi T, Soghli N. The regulatory functions of circular RNAs in osteosarcoma. Genomics. 2020;112(4):2845-56. doi: 10.1016/j.ygeno.2020.03.024. [PubMed: 32243895].

14. Mohammadzadeh I, Qujeq D, Yousefi T, Ferns GA, Maniati M, Vaghari-Tabari M. CRISPR/Cas9 gene editing: A new therapeutic approach in the treatment of infection and autoimmunity. IUBMB Life. 2020;72(8):1603-21. doi: 10.1002/iub.2296. [PubMed: 32344465].

15. Li M, Song L, Qin X. Glycan changes: cancer metastasis and anti-cancer vaccines. J Biosci. 2010;35(4):665-73. doi: 10.1007/s12038-010-0073-8. [PubMed: 21289447].

16. Ferreira JA, Magalhaes A, Gomes J, Peixoto A, Gaiteiro C, Fernandes E, et al. Protein glycosylation in gastric and colorectal cancers: Toward cancer detection and targeted therapeutics. Cancer Lett. 2017;387:3245. doi: 10.1016/j.canlet.2016.01.044. [PubMed: 26828132].

17. Glavey SV, Huynh D, Reagan MR, Manier S, Moschetta M, Kawano Y, et al. The cancer glycome: carbohydrates as mediators of metastasis. Blood Rev. 2015;29(4):269-79. doi: 10.1016/j.blre.2015.01.003. [PubMed: 25636501].

18. Miyoshi E, Moriwaki K, Terao N, Tan CC, Terao M, Nakagawa T, et al Fucosylation is a promising target for cancer diagnosis and therapy. Biomolecules. 2012;2(1):34-45. doi: 10.3390/biom2010034. [PubMed: 24970126]. [PubMed Central: PMC4030867].

19. Blanas A, Sahasrabudhe NM, Rodriguez E, van Kooyk Y, van Vliet SJ. Fucosylated antigens in cancer: An alliance toward tumor progression, metastasis, and resistance to chemotherapy. Front Oncol. 2018;8:39. doi: 10.3389/fonc.2018.00039. [PubMed: 29527514]. [PubMed Central: PMC5829055].

20. Yan X, Lin Y, Liu S, Aziz F, Yan Q. Fucosyltransferase IV (FUT4) as an effective biomarker for the diagnosis of breast cancer. Biomed Pharmacother. 2015;70:299-304. doi: 10.1016/j.biopha.2014.12.048. [PubMed: 25776515].

21. Li Y, Sun Z, Liu B, Shan Y, Zhao L, Jia L. Tumor-suppressive miR-26a and miR-26b inhibit cell aggressiveness by regulating FUT4 in colorectal cancer. Cell Death Dis. 2017;8(6). e2892. doi: 10.1038/cddis.2017.281. [PubMed: 28640257]. [PubMed Central: PMC5520934].

22. Sohrabi M, Gholami A, Azar MH, Yaghoobi M, Shahi MM, Shirmardi S, et al. Trace element and heavy metal levels in colorectal cancer: Comparison between cancerous and non-cancerous tissues. Biol Trace Elem Res. 2018;183(1):1-8. doi: 10.1007/s12011-017-1099-7. [PubMed: 28795369]. 
23. Bo S, Durazzo M, Gambino R, Berutti C, Milanesio N, Caropreso A, et al Associations of dietary and serum copper with inflammation, oxidative stress, and metabolic variables in adults. J Nutr. 2008;138(2):30510. doi: 10.1093/jn/138.2.305. [PubMed: 18203896].

24. Khoshdel Z, Naghibalhossaini F, Abdollahi K, Shojaei S, Moradi M, Malekzadeh M. Serum copper and zinc levels among Iranian colorectal cancer patients. Biol Trace Elem Res. 2016;170(2):294-9. doi: 10.1007/s12011-015-0483-4. [PubMed: 26329996].

25. Gupte A, Mumper RJ. Elevated copper and oxidative stress in cancer cells as a target for cancer treatment. Cancer Treat Rev. 2009;35(1):3246. doi: 10.1016/j.ctrv.2008.07.004. [PubMed:18774652]

26. Skrovanek S, DiGuilio K, Bailey R, Huntington W, Urbas R, Mayilvaganan B, et al. Zinc and gastrointestinal disease. World J Gastrointest Pathophysiol. 2014;5(4):496-513. doi: 10.4291/wjgp.v5.i4.496. [PubMed: 25400994]. [PubMed Central: PMC4231515].

27. Taniguchi N, Kizuka Y, Takamatsu S, Miyoshi E, Gao C, Suzuki K, et al. Glyco-redox, a link between oxidative stress and changes of glycans: Lessons from research on glutathione, reactive oxygen and nitrogen species to glycobiology. Arch Biochem Biophys. 2016;595:72-80. doi: 10.1016/j.abb.2015.11.024. [PubMed: 27095220].

28. Osuga T, Takimoto R, Ono M, Hirakawa M, Yoshida M, Okagawa Y, et al. Relationship between increased fucosylation and metastatic potential in colorectal cancer. I Natl Cancer Inst. 2016;108(8). doi: 10.1093/jnci/djw038. [PubMed: 27075853]. [PubMed Central:
PMC5017955]

29. Yazawa S, Madiyalakan R, Izawa H, Asao T, Furukawa K, Matta KL. Cancer-associated elevation of alpha(1-3)-L-fucosyltransferase activity in human serum. Cancer. 1988;62(3):516-20. doi:10.1002/10970142(19880801)62:3<516::aid-cncr2820620313>3.0.co;2-4. [PubMed: 3390792].

30. Muinelo-Romay L, Vazquez-Martin C, Villar-Portela S, Cuevas E, GilMartin E, Fernandez-Briera A. Expression and enzyme activity of alpha(1,6)fucosyltransferase in human colorectal cancer. Int I Cancer. 2008;123(3):641-6. doi:10.1002/ijc.23521. [PubMed:18491404].

31. Muinelo-Romay L, Villar-Portela S, Cuevas Alvarez E, Gil-Martin E, Fernandez-Briera A. alpha(1,6)Fucosyltransferase expression is an independent prognostic factor for disease-free survival in colorectal carcinoma. Hum Pathol. 2011;42(11):1740-50. doi: 10.1016/j.humpath.2011.01.021. [PubMed: 21652057].

32. Al Faris NA, Ahmad D. Distribution of trace elements like calcium, copper, iron and zinc in serum samples of colon cancer A case control study. J King Saud Univ Sci. 2011;23(4):337-40. doi: 10.1016/j.jksus.2010.07.017.

33. Stepien M, Jenab M, Freisling H, Becker NP, Czuban M, Tjonneland A, et al. Pre-diagnostic copper and zinc biomarkers and colorectal cancer risk in the European Prospective Investigation into Cancer and Nutrition cohort. Carcinogenesis. 2017;38(7):699-707. doi: 10.1093/carcin/bgx051. [PubMed: 28575311]. 